\title{
Cribado del cáncer de cérvix: ¿la misma frecuencia para mujeres inmigrantes latinoamericanas?
}

Y. González Rubio, M. S. Castaño Pinto

Especialistas en Medicina Familiar y Comunitaria.

Centro de Salud Monóvar. Madrid

\section{RESUMEN}

Debido al cambio demográfico que está viviendo nuestro país con la llegada de inmigrantes de dife rentes nacionalidades, nos preguntamos si estos nuevos usuarios del Sistema Nacional de Salud pre sentan los mismos o diferentes problemas de salud que la población autóctona. En particular en este artículo nos planteamos si las mujeres provenientes de América del Sur, población mayoritaria de inmi grantes en nuestra zona de salud, presentan diferen te riesgo de padecer cáncer de cuello uterino, y por ello precisan cambios en el programa de cribado. Buscando información en la literatura no hemos en contrado evidencias de que sea necesario variar los intervalos en el programa de screening, pese a que existen diferencias en la prevalencia y en algunos factores de riesgo para esta enfermedad.

Palabras clave: Cáncer de cuello. Cribado. Población latinoamericana.
Screening for cervix cancer: same incidence in latinoamerican immigrants women?

\begin{abstract}
Due to the demographic change that our country is living with the arrival of immigrants of different nationalities, we wonder if these new users of the National System of Health present the same or diffe rent problems of health that the autochthonous po pulation. In particular in this article we think about if the women coming from America of the South, im migrants' majority population in our area of health, they present different risk of suffering cancer of ute rine cervical, and for they specify it changes in the screening program. Looking for information in the literature has not found evidences that it is neces sary to vary the intervals in the screening program, in spite of the fact that differences exist in the preva lence and in some factors of risk for this illness.
\end{abstract}

Key words: Cervical cancer. Screening. Latin American population.

\section{INTRODUCCIÓN}

El cáncer de cuello de útero es el tumor más frecuente entre las mujeres de países en desarrollo y el segundo en el ámbito mundial. La incidencia de este tumor varía mucho, correspondiendo a América Latina la tasa más alta y a España una de las incidencias más bajas.

El tipo histológico de mayor importancia epidemiológica es el carcinoma escamoso que se origina en el epitelio de transición del cuello uterino. Hay diversos factores implicados en la génesis de este tumor siendo el virus del papiloma humano (HPV) el mayor factor de riesgo.

La lenta evolución del tumor, la accesibilidad anatómica y la existencia de una prueba de detección de lesiones precursoras, la citología exfoliativa, parecen haber influido en la extensión de los programas de cribado de cáncer de cérvix como medida de prevención secundaria. La re- 
ducción de la mortalidad por este cáncer depende de la cobertura que alcanza el programa, de la periodicidad y de la edad de inicio y fin de las citologías.

La efectividad y eficiencia de este programa de detección precoz están relacionadas con la incidencia del tumor, la historia natural de la enfermedad, la validez de la citología y las dificultades en la captación de los grupos de mayor riesgo y por tanto de mayor incidencia. En España, la baja frecuencia del tumor ocasiona un bajo valor predictivo positivo y un bajo rendimiento del progra$\mathrm{ma}^{1}$. Cuando se han aplicado los programas en otros países con incidencias más altas o en mujeres con más factores de riesgo, la efectividad ha sido mayor ${ }^{2}$.

Ante el aumento en nuestra zona de salud de mujeres de origen latinoamericano, procedentes de lugares con mayor incidencia de cáncer de cérvix, nos planteamos si la aplicación del programa de detección entre ellas debe de modificarse en cuanto al inicio o la periodicidad de la prueba.

\section{MAGNITUD DEL PROBLEMA: INCIDENCIA Y MORTALIDAD}

La incidencia del cáncer de cérvix uterino es mayor en países en vías de desarrollo ${ }^{3}$, incluidos América Latina (que presenta la mayor incidencia mundial, 55 casos por cada 100.000 mujeres $^{4}$ y el Caribe según datos de 1982). La incidencia del cáncer en España es de 4-10 casos por cada 100.000 mujeres $^{1,5}$ (1983-1985). Hay una mayor incidencia entre los 20 y los 40 años de lesiones premalignas y por encima de los 50 años de lesiones malignas. Se ha observado un aumento de lesiones malignas en mujeres más jóvenes, debido probablemente a un aumento en el número de diagnósticos de estas lesiones.

Las curvas de incidencia para el cáncer de cérvix siguen el mismo patrón en casi todos los países: a partir de un determinado punto aparece un ascenso rápido hasta alcanzar un pico y luego un descenso muy lento o una meseta ${ }^{2}$. El pico máximo es lo que cambia en las distintas localizaciones: en el Norte de Europa es a los 44-49 años y otro pequeño pico a los 70-79 años, en el resto de Europa, América, Asia y África el pico máximo es a los 50-65 años (Fig. 1). La mortalidad por esta enfermedad no sigue necesariamente estos patrones de incidencia. Así, aunque la incidencia de cáncer de cérvix es mayor en EE.UU. que en los países de la Unión Europea, la mortalidad es menor. En España la tasa de mortalidad fue de 2,7 por 100.000 mujeres en $1994^{5}$, y en el resto de Europa

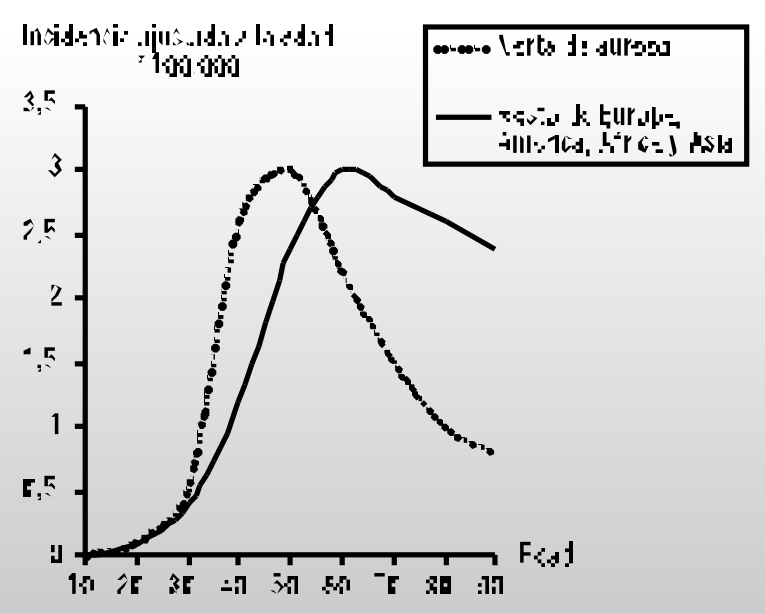

Figura 1

Distribución etaria de la frecuencia de cáncer de cérvix. Modificado de Gustaffson', 1997.

oscila entre 2,9 por 100.000 mujeres en Holanda y 5,5 por 100.000 mujeres en Dinamarca, según cifras de 1989-19907 . La tendencia temporal de incidencia y mortalidad en Europa es descendente. En América del Sur la tasa de mortalidad es más alta, oscilando entre 11,9 por 100.000 mujeres de Chile hasta 2,8 por 100.000 mujeres en Puerto Rico (situándose dicha tasa en el resto de los países en torno a 5-6 por 100.000 mujeres), datos obtenidos en $1980^{8}$.

\section{FACTORES DE RIESGO PARA EL CÁNCER DE CÉRVIX}

Distintos elementos han sido implicados como factores de riesgo en el desarrollo del cáncer de cérvix, y el virus del papiloma humano es el factor de riesgo más importante según la evidencia científica para la aparición de la enfermedad, especialmente algunos subtipos, como el 16, $18 \mathrm{y}$ en menor medida 35,31 y 33 . Este virus, que se transmite con las relaciones sexuales, una vez que contacta con el cérvix uterino es capaz de contribuir a la aparición de neoplasias por la presencia en su DNA de oncogenes. La aparición del virus se relaciona, como el resto de enfermedades de transmisión sexual (ETS), con el elevado número de parejas sexuales (tanto de las mujeres como de sus compañeros) y la no-utilización de preservativo.

La prevalencia de la infección por HPV también es diferente según los países. En América del Sur y Central aparecen con frecuencia otros subtipos de HPV, subtipos 18, 45, 31 y 33, pero tanto en estas 
zonas como en Europa el más prevalente es el subtipo 16. En un estudio casos-controles realizado en España y Colombia los datos de prevalencia de infección por HPV fueron del $41,7 \%$ en Colombia y del $42,6 \%$ en España.

La edad de inicio de las relaciones sexuales también ha demostrado ser factor de riesgo para el desarrollo de cáncer de cérvix. Parece que su influencia se debe al daño ocasionado por la estimulación del epitelio del cuello del útero todavía en desarrollo, o a la mayor exposición (durante más tiempo) a la infección por HPV. Algunos estudios han demostrado que es factor de riesgo el inicio precoz de las relaciones por debajo de los 16 años y otros estudios sitúan esta edad por debajo de los 20 años. No está claro por qué el pertenecer a un bajo nivel socioeconómico es un factor de riesgo ${ }^{10}$. Algunas posibilidades que se han planteado en la literatura son: un menor número de screening solicitados, el inicio más precoz de las relaciones sexuales entre estas mujeres o una mayor tasa de fumadoras en este estatus. En algunos estudios se ha llegado a hablar de sinergismo entre varios de estos factores.

Otro factor de riesgo es el número de parejas sexuales. Aunque la presencia de infección por HPV se relaciona con el número de parejas sexuales y con la presencia de cáncer, hay estudios que demuestran que existe relación entre un alto número de parejas sexuales (mayor de 6) y cáncer de cérvix, incluso entre mujeres con test HPV negativo ${ }^{10}$. Esto puede deberse a que sea un factor independiente o a la infradetección de la infección por HPV e incluso a la presencia de otras ETS, por ejemplo la infección por Chlamy dia trachomatis, que también ha demostrado relación con el aumento de incidencia del cáncer de cérvix ${ }^{13}$.

Unos estudios han demostrado relación entre un carcinógeno del tabaco (el NKK) y la aparición del cáncer de cérvix ${ }^{14}$ y otros lo desmienten como factor de riesgo ${ }^{9}$. Y al igual que ocurre con el tabaco, no existe claro acuerdo en la literatura si los anti conceptivos orales (ACOs), especialmente desde la aparición de ACOs con menores dosis de estrógenos y gestágenos, puede influir en la aparición de este cáncer. Algunos estudios han demostrado que la utilización de ACOs en mujeres con infección por HPV durante más de 10 años sí podría ser un factor de riesgo ${ }^{10}$.

Otros factores son el cáncer de pene en la pareja, la inmunodepresión y VIH. También parece ser factor de riesgo el antecedente de otras ETS distintas del HPV, especialmente Chlamydia trachoma tis, y Neisseria gonorrheae y herpes virus tipo II. No queda claro si estas enfermedades son factor de riesgo en sí mismas o marcadores de infradetección de infección por $\mathrm{HPV}^{13}$.

\section{PREVENCIÓN SECUNDARIA}

La detección precoz del cáncer de cérvix es fácilmente realizable por la larga evolución de la enfermedad que pasa por distintos estadios (displasia, cáncer in situ y cáncer invasivo) y por la existencia de una técnica (la citología exfoliativa o de Papanicolau) para realizar esta detección precoz.

La realización de citología cervicovaginal como método de cribado del cáncer de cérvix, es probablemente la mejor forma de prevención secundaria de que disponemos actualmente para esta enfermedad. Pese a que en algunos países (especialmente del Norte de Europa) hay descensos claros de la incidencia y la mortalidad desde la implantación de esta medida, en otros países, como Gran Bretaña ${ }^{15}$, no ha sucedido lo mismo hasta los años noventa, cuando se consiguió un aumento en la cobertura. Hasta ahora, la evidencia científica existente de estudios controlados randomizados es pequeña, ya que desde la implantación de este método de cribado, no sería muy ético la realización de dichos estudios. Los datos disponibles en la actualidad se han obtenido de estudios experimentales y de tendencias temporales ${ }^{6,16}$

La citología exfoliativa es una prueba con una especificidad alta $(90 \%)$ y con una sensibilidad que varía según los autores entre el 55 al 90\% ${ }^{17}$, lo que limita su validez. La proporción de falsos positivos y negativos del Papanicolau va a depender mucho de la técnica de toma de las muestras, del procesamiento y de la lectura de éstas ${ }^{18}$, según el IARC ${ }^{7}$ los falsos positivos y negativos se sitúan en torno al $40 \%$. De los resultados falsos negativos, en torno a una tercera parte se atribuye a errores en la lectura e interpretación y el resto a fallos en la toma de las muestras ${ }^{1}$, que se mejoraría con el entrenamiento específico de las personas encargadas de la toma y la lectura.

Otras limitaciones de la validez de la prueba en nuestro país serían la baja incidencia de este tumor y las dificultades para lograr altas tasas de participación y hacer llegar el programa a los grupos de mayor riesgo.

La citología cervical aunque mínimamente invasiva puede ocasionar efectos adversos en las mujeres como molestias físicas y psicológicas. Derivadas de resultados falsos positivos y negativos, reexploraciones para confirmar el diagnóstico, intervenciones sanitarias innecesarias y ansiedad en las mujeres detectadas como positivas, con el consiguiente aumento en los costes del programa.

No existe acuerdo entre las distintas sociedades médicas sobre cuál es el intervalo más adecuado para la realización de cribado del cáncer de cérvix ni el rango etario que debe abarcar. En la 
actualidad se desconoce cuál es el intervalo más coste-efectivo. Según las distintas sociedades nos encontramos con recomendaciones diversas: la Sociedad Americana del Cáncer, el Colegio Americano de Obstetras y Ginecólogos y la Academia Americana de Médicos de Familia recomiendan que el inicio del cribado sea con el inicio de las relaciones sexuales o a los 18 años, una periodicidad al inicio anual (durante 3 años) y si éstas son normales puede hacerse con menor frecuencia en mujeres de bajo riesgo y a criterio del médico; no ponen límite superior de edad para dejar de hacer citología. La Canadian Task Force recomienda el mismo inicio, realizarlas cada 3 años hasta los 35 y cada 5 hasta los 75 años. La Guía Europea (Programa Europa Contra el Cáncer) plantea un intervalo etario de 25 a 60 años, con un intervalo de 3 años entre cada citología. El PAPPS $^{1}$ recomienda que el cribado se realice en mujeres sexualmente activas mayores de 35 años y menores de 65 años, con una periodicidad anual durante los dos primeros años y posteriormente con intervalos de 5 años, si las previas son normales. A mujeres mayores de 65 años (sin citología en los anteriores 5 años), dos citologías separadas un año y si son normales, no se harán más intervenciones. En todas estas recomendaciones se contempla que si hay citologías patológicas o factores de riesgo la periodicidad puede aumentar a criterio del médico (Tabla I).

Los motivos de considerar esos intervalos etarios y esas frecuencias de realización de citologías, son controvertidos y diversos según los artículos consultados. El inicio del cribado coincidiendo con el inicio de las relaciones se- xuales o a los 18 años tiene su explicación en que se ha demostrado que sólo las mujeres sexualmente activas, o que lo han sido, presentan riesgo de sufrir cáncer de cérvix y en que dos tercios de la población americana ${ }^{17}$ y el $75 \%$ de la colombiana $^{10}$ ya es sexualmente activa a los 18 años. Sin embargo, el PAPPS no recomienda el inicio del cribado hasta los 35 años porque se conoce que aproximadamente un $84 \%$ de las lesiones premalignas en mujeres menores de esa edad no progresan $^{19}$; aunque según otros estudios está aumentando la incidencia de cáncer de cérvix invasivo en mujeres jóvenes ${ }^{4}$. No queda claro según la evidencia cuál es la edad adecuada de inicio del cribado, lo que explica las diferencias entre las recomendaciones de los grupos de expertos.

En relación con la exclusión del cribado a los 65 años parece demostrado que si las citologías previas son normales es raro que vaya a aparecer la enfermedad a esa edad, pero siempre es necesario tener al menos dos negativas para excluir. Si la paciente nunca acudió al screening hay que realizar citologías al menos durante dos años seguidos y luego excluir del programa.

Hay acuerdo unánime en que la frecuencia inicial sea anual (durante dos o tres años) pese a que no existe evidencia de que esto disminuya la incidencia o la mortalidad del cáncer de cérvix ${ }^{15}$. La explicación es sencilla ya que así se garantiza la disminución de falsos positivos y negativos que se pueden presentar en una prueba única.

Por último, la frecuencia de realización de la citología es igualmente un motivo de discusión. Los autores creen que el efecto sobre la incidencia varía según los distintos intervalos de cribado. Day opina

\begin{tabular}{|c|c|c|c|}
\hline \multicolumn{4}{|c|}{ RECOMENDACIONES DE SCREENING } \\
\hline $\begin{array}{l}\text { SOCIEDAD } \\
\text { MÉDICA }\end{array}$ & INICIO DEL CRIBADO & PERIODICIDAD & FIN DEL CRIBADO \\
\hline $\begin{array}{l}\text { Sociedad Americana } \\
\text { del Cáncer }\end{array}$ & $\begin{array}{l}\text { Inicio de relaciones o } 18 \text { años, } \\
\text { anual durante } 3 \text { años }\end{array}$ & A criterio médico & No hay límite \\
\hline $\begin{array}{l}\text { Canadian Task } \\
\text { Force }^{23}\end{array}$ & $\begin{array}{l}\text { Inicio de relaciones o } 18 \text { años, } \\
\text { anual durante } 3 \text { años }\end{array}$ & $\begin{array}{l}\text { Trianual hasta los } 35 \\
\text { años. Quinquenal entre } \\
\text { 35-75 años }\end{array}$ & 75 años \\
\hline $\begin{array}{l}\text { Guía Europea contra } \\
\text { el cáncer }\end{array}$ & Inicio a los 25 años & Trianual o quinquenal & 60 años \\
\hline PAPPS $^{1}$ & $\begin{array}{l}\text { Dos test anuales en mujeres } \\
\text { sexualmente activas (desde } \\
\text { los } 35 \text { años) }\end{array}$ & Quinquenal & 65 años \\
\hline
\end{tabular}


que haciendo citología anual desde los 20 a los 64 años conlleva una reducción de la incidencia de cáncer de un $93,3 \%$, frente a la reducción del $91,2 \%$ con citología trianual y del $83,8 \%$ con citología quinquenal (disminuyendo en 20 y en 27 el número de citologías a lo largo de la vida de la mujer). Herrero et $\mathrm{al}^{2}$, en un estudio de casos controles no encuentra diferencias significativas en el riesgo relativo de presentar cáncer de cérvix entre los intervalos de 12 a 23 meses frente a intervalos de 24 a 47 meses, y sí que hay un riesgo mayor por encima de 48 meses (RR 1-2,5).

Otro factor a tener en cuenta, y de gran importancia, es a cuántas mujeres se llega con las campañas de cribado del cáncer de cérvix. En España, según datos del Programa Europa contra el cáncer $(1987)^{21}$, sólo un $12 \%$ de las mujeres se realiza citología frente al $40 \%$ de Italia o el $60 \%$ de Francia. Además a este cribado acuden preferentemente las mujeres con menores factores de riesgo, mujeres con alto nivel sociocultural o jóvenes, que son las que más utilizan los servicios de planificación familiar, ginecológicos o servicios postnatales. Sin embargo, las mujeres de mayor edad, por encima de 50 años (que son las que tienen más riesgo de cáncer invasivo) y con bajo nivel sociocultural, son las que menos citologías se realizan, probablemente por miedo o vergüenza y por menor comprensión de la importancia de realizarse el cribado ${ }^{2,17}$.

\section{DISCUSIÓN Y COMENTARIOS}

La llegada de inmigrantes, principalmente de origen latinoamericano, a nuestra zona de Salud, que acudían a solicitar su revisión ginecológica como motivo de consulta aparentemente más frecuente, nos hizo plantearnos si estas mujeres tenían factores de riesgo diferentes a las mujeres españolas, para cáncer de cérvix y qué tipo de seguimiento debía de realizarse con ellas.

La incidencia de la enfermedad es 10 veces mayor en Latinoamérica que en España, y según los estudios revisados sólo la edad de inicio de las relaciones y el número de parejas sexuales difieren entre ambas poblaciones ${ }^{10,13}$. La prevalencia de HPV, principal factor de riesgo, es similar entre mujeres latinoamericanas y españolas ${ }^{9}$. Y tanto en España como en Latinoamérica el grupo de mujeres con mayor prevalencia de cáncer es aquel al que menos llega el programa de detección, e incluye las mujeres de más edad, las que tienen un nivel de educación más bajo, las mujeres sin enfermedad de transmisión sexual y las que nunca usaron ACOs, (éstos dos últimos grupos por no recibir información adecuada al no haber acudido a los servicios médicos) ${ }^{2}$.
$¿$ Tenemos datos para indicar unos intervalos determinados a estas mujeres? Para Herrero et $\mathrm{al}^{2}$ no hay diferencias estadísticamente significativas entre realizar citologías con intervalo de 12 a 23 meses frente al intervalo de 24 a 47 meses (en el estudio realizado entre población colombiana y española) y para Day $^{20}$ tampoco las hay entre citologías anuales y trianuales. Según este mismo autor el hacer citologías trianuales en vez de anuales, disminuye en 20 el número de citologías que una mujer se hace a lo largo de su vida. Parece necesario llegar a un equilibrio entre la necesidad del cribado para reducir la incidencia de cáncer invasivo y la realización de un número excesivo de citologías que tampoco parece aceptable para la población, en gran parte por factores socioculturales.

Existe controversia en la edad de inicio del programa de detección precoz. El grupo de expertos del PAPPS no aconseja iniciar el screening hasta los 35 años, y sin embargo otras sociedades lo aconsejan a los 18, dada la precocidad sexual de su población. No queda claro según la evidencia científica cuál es la edad adecuada de inicio del cribado, lo que explica las diferencias entre las recomendaciones de los grupos de expertos.

Dado que en España la incidencia de cáncer de cérvix es muy baja (5,7 a 7,4 de cada 100.000 mujeres), el descenso de incidencia que se puede conseguir tras la aplicación del programa, si participara el $100 \%$ de la población, sería aproximadamente de 5,1 casos por 100.000 mujeres con el intervalo de screening cada cinco años, de 5,4 en el intervalo trianual y de 5,5 en el intervalo anual. En Latinoamérica (con una incidencia de 55 casos por 100.000 mujeres) el descenso conseguido sería 51, 50 y 46, respectivamente (Tabla II).

\begin{tabular}{|c|c|c|c|}
\hline \multicolumn{4}{|c|}{$\begin{array}{ccc}\text { EFECTIVIDAD DE ESTRATEGIAS DE SCREENING } & \text { Tabla II }\end{array}$} \\
\hline $\begin{array}{l}\text { INTERVALO ENTRE } \\
\text { CITOLOGÍAS } \\
\text { (AÑOS) }\end{array}$ & $\begin{array}{l}\text { GRUPO DE } \\
\text { EDAD }\end{array}$ & $\begin{array}{l}\text { REDUCCIÓN } \\
\text { INCIDENCIA \% }\end{array}$ & $\begin{array}{l}\text { NÚMERO DE } \\
\text { CITOLOGÍAS }\end{array}$ \\
\hline 1 & $20-64$ & 93 & 45 \\
\hline 3 & $20-64$ & 91 & 15 \\
\hline 5 & $20-64$ & 84 & 9 \\
\hline
\end{tabular}

\section{RECOMENDACIONES FINALES}

Una de las recomendaciones de la estrategia del PAPPS para el cáncer de cérvix es la búsqueda ac- 
tiva de las mujeres con factores de riesgo y de igual importancia consideramos fomentar la participación de la población, que debe sistematizarse desde la Atención Primaria de Salud.

Según la literatura revisada, no parece necesario acortar los periodos de realización de las citologías en mujeres inmigrantes, sino mantener la periodicidad trianual. Y con relación al inicio del programa parece adecuado seguir los consejos de las recomendaciones internacionales a favor del inicio a los 18 años.

Más allá de la Atención Primaria, se deben aplicar criterios de mejora en la técnica de toma de muestras, procesamiento y lectura, en la emisión de los informes y en la evaluación continuada del programa.

No podemos concluir sin recordar que la educación para la salud es la opción más indicada en la prevención primaria del cáncer de cérvix. Es necesaria la difusión de información sobre la uti- lización del preservativo para reducir la incidencia de enfermedades de trasmisión sexual, la promoción de hábitos sexuales saludables y de campañas antitabaco y de deshabituación tabáquica. Hay que incidir especialmente en los grupos de población más desfavorecida (estatus socioeconómico más bajo) o con mayor riesgo (adolescentes).

\section{CORRESPONDENCIA:}

Yolanda González Rubio Centro de Salud Monóvar

C/ Monóvar $\mathrm{n}^{\circ} 11$

28033 Madrid

Telf.: 913838989

\section{Bibliografía}

1. Alonso JM, Bellas B, Cierco P, Gálvez M, González J, Marín N, et al. Prevención del cáncer. Atención primaria 1999; 24 (S1): 83-6.

2. Herrero R, Brinton LA, Reeves WC, Brenes MM, de Britton RC, Gaitan E, et al. Screening for cervical cancer in Latin America: A case-control study. Int J Epidemiol 1992; 21: 1050-6.

3. Weston R, Sheperd J, Napuli Z. Interventions for encouraginig sexual lifestyles and behaviours intended to prevent cervical cancer (Protocol for a Cochrane Review). In: The Cochrane Library, Issue 1, 1999. Oxford: Update Software.

4. Jensen OM, Estève J, Moller H, Renard H. Cancer in the European Community and its members states. Eur J Cancer 1990; 26: 1167-256.

5. Rivas A, Salcedo A. Cáncer ginecológico. En Martin Zurro A, Cano Pérez JF, editores. Atención Primaria. Conceptos, organización y práctica clínica. Barcelona: Doyma SA, 1994; 910-24.

6. Gustafsson L, Pontén J, Bergströn R, Adami HO. International incidence rates of invasive cervical cancer before cytological screening. Int J Cancer 1997; 71: 159-65.

7. Estéve J, Kricker J, Ferlay J, Parkin DM, eds. Facts and figures of cancer in tue European Community. Lyon: IARC, 1993.

8. Restrepo H, González J, Roberts E, Litvak J. Epidemiología y control del cáncer de cuello uterino en América Latina y el Caribe. Bol of Sanit Panam 1987; 102(6): 578-92.

9. Bosch FX, Manos MM, Muñoz N, Sherman M, Jansen AJ, Schiffman MH, et al. Prevalence of Human Papillomavirus in Cervical Cancer: a Worldwide Perspective. Natl Cancer Inst 1995; 87: 796-802.
10. Bosch FX, Muñoz N, De Sanjosé S, Izarzugaza I, Gili M, Viladiu $\mathrm{P}$ et al. Risk Factors for Cervical Cancer in Colombia and Spain. Int J Cancer 1992; 52: 750-8.

11. Wellings K, Field J, Johnson A, Wadswoth J. Sexual behaviour in Britain - the national survey of sexual attitudes and lifestyles. Penguin, England, 1994.

12. Buitrago F, Vergeles-Blanca JM, Cardeñosa G. Cáncer de cuello uterino. FMC 1997; 4: 292-300.

13. De Sanjose S, Muñoz N, Bosch FX, Reimann K, Pedersen NS, Orfila J, et al. Sexually transmitted agents and cervical neoplasia in Colombia and Spain. Int J Cancer 1994; 56: 358-63.

14. Prokopczyk B, Cox JE, Hoffman D, Waggoner SE. Identification of tobacco-specific carcinogen in the cervical mucus of smokers and non-smokers. J Natl Cancer Inst 1997; 89: 868-73.

15. Martin J, Mínguez JA, Otero JA. Detección precoz del cáncer de cuello de útero. FMC 1995; 2: 249-56.

16. Sigurdson K, Adalsteinsson S, Ragnarsson J. Trends in cervical and breast cancer in Iceland. A statistical evaluation of trends in incidence and mortality for the period 1955-1989. Their relation to screening and prediction to the year 2000 . Int J Cancer 1991; 48: 523-8.

17. Borrás JM, Iglesias X. Cribado del cáncer de cuello de útero Med Clin (Barc) 1994; 102 Suppl 1:80-84.

18. Failures of Cervical Cancer Screening (editorial). American Journal of Public Health 1995; vol 85, n6: 61-762.

19. Oortmarssen GJ, Habema JDF. Epidemiological evidence for age-dependent regression or pre-invasive cervical cancer. Br J Cancer 1991; 64: 559-65. 
20. Day NE. Screening for cancer of cervix. J Epidemiol Community Health 1989; 43: 103-6.

21. Programa Europa contra el cáncer. Los europeos y la prevención del cáncer. Bruselas: Comunidad Económica Europea, 1987.

22. Updated January 1992. The American Cancer Society guidelines for the cancer-related checkup. CA Cancer J Clin 1992; 42: 44-5.
23. Jonson K. Periodic health examination, 1995 update: 1, Screening for human papillomavirus infection in asymtomatic women. Canadian Task Force on the Periodic Health Examination. Can Med Assoc J 1995; 152: 483-93.

24. Coleman D, Day N, Douglas G, Farmery E, Lynge E, Philip J, Segnan N. European Guidelines for Quality Assurance in Cervical Cancer Screening. Eur J Cancer 1993; 29: 1-38. 